\title{
Stochastic Path Perturbation Approach Applied to Non-Local Non-Linear Equations in Population Dynamics
}

\author{
M.A. Fuentes ${ }^{1,2,3}$, M.O. Cáceres ${ }^{4}$ * \\ ${ }^{1}$ Santa Fe Institute, 1399 Hyde Park Road, Santa Fe, New Mexico 87501, USA \\ ${ }^{2}$ Instituto de Investigaciones Filosóficas, Bulnes 642, 1428 Buenos Aires, Argentina \\ ${ }^{3}$ Universidad San Sebastián, Lota 2465, Santiago 7500000, Chile \\ ${ }^{4}$ Centro Atómico Bariloche, Instituto Balseiro and CONICET, 8400 Bariloche, Argentina
}

\begin{abstract}
We described the first passage time distribution associated to the stochastic evolution from an unstable uniform state to a patterned one (attractor of the system), when the time evolution is given by an integro-differential equation describing a population model. In order to obtain analytical results we used the Stochastic Path Perturbation Approach introducing a minimum coupling approximation into the nonlinear dynamics, and a stochastic multiscale perturbation expansion. We show that the stochastic multiscale perturbation is a necessary tool to handle other problems like: nonlinear instabilities and multiplicative stochastic partial differential equations. A small noise parameter was introduced to define the random escape of the stochastic field. We carried out Monte Carlo simulations in a non-local Fisher like equation, to show the agreement with our theoretical predictions.
\end{abstract}

Keywords and phrases: non-linear population dynamics, non-local logistic models, Fisher equation, random escape times, first passage time distribution

Mathematics Subject Classification: 35R9, 35R60, 92D25

\section{Introduction}

In the last twenty years the study of non-local models in ecology and biology has led to much interest, most of them have been formulated in terms of continuous-field evolution equations for densities describing long distance interactions [1-3], but also in terms of discrete master evolution equations [4]. These interactions can be mediated through vision, hearing, smelling or other kind of sensing. Therefore, non-local effects in the non-linear terms in reaction-diffusion equations may account for resource's competition within a certain range. Works quoting the importance of these type of dynamical models can be found in the present issue. It is worth mentioning studies of bacteria cultures on petri dishes, in which the diffusion of nutrients and/or the release of toxic substances can cause non-locality in the interactions [5-8]. Moreover, we can mention related works, as the study of travelling wave solutions of non-local reaction-diffusion equations arising also in population dynamics [9]. Other study refer to the pattern formation phenomena

${ }^{*}$ Corresponding author. E-mail: caceres@cab.cnea.gov.ar

(C) EDP Sciences, 2015 
in a model of competing populations with non-local interactions [10], and bifurcation theory [11,12]. There are also some pieces of work related to neural fields, where non-local interactions and noise-induced jumps play an important role in the description of real systems $[13,14]$.

In this contribution we focus on the study of a stochastic non-local (integro-differential) version of the so-called Lotka-Volterra, or Fisher, equation $[3,15,16]$. We are specially concerned on the description of the random escape time of the field system -from a uniform stationary to a patterned state near criticality- introducing a stochastic multiscale perturbation expansion.

In the next section we show the mathematical model used in this study, next we perform the Fourier analysis of the non-local model. In section IV we introduce the stochastic multiscale perturbation expansion, and finally in section $\mathrm{V}$ we introduce our discussions and future work.

\section{Mathematical Model}

The dynamical model, shown in the next equation, takes into account the exponential growth of the population, characterized by the parameter $a$, a diffusion constant $D$, a non-local competition term, proportional to a parameter, $b$, and the kernel $G(x)$.

We also model environmental/thermic fluctuations acting on these types of systems. To take this into account, we introduce an additive fluctuating Gaussian field $\xi(x, t)$ in the dynamics. This is a plausible ansatz when the unspecified random contributions are more important at low density, see Appendix 3 in [17]. We characterized the strength of the noise with a small parameter $\epsilon$.

Then, the one dimensional model looks as follows

$$
\frac{\partial u(x, t)}{\partial t}=D \frac{\partial^{2} u(x, t)}{\partial x^{2}}+a u(x, t)-b u(x, t) \int_{\mathcal{D}} u\left(x-x^{\prime}, t\right) G\left(x^{\prime}\right) d x^{\prime}+\sqrt{\epsilon} \xi(x, t) .
$$

We are interested in the stochastic pattern formation description of the (positive) density field $u(x, t)$ of Eq.(2.1), subject to periodic boundary conditions on $\mathcal{D}=[-L, L]$. The random characteristics of this stochastic integro-differential equation is completely characterized by the statistics of the field $\xi(x, t)$. Nevertheless the first passage time problem associated to this model is non-trivial, due to the characteristics introduced by the non-local term contribution. In the present study we included Gaussian white-noise moments $[18-20]$

$$
\langle\xi(x, t)\rangle=0 ; \quad\left\langle\xi(x, t) \xi\left(x^{\prime}, t^{\prime}\right)\right\rangle=\delta\left(x-x^{\prime}\right) \delta\left(t-t^{\prime}\right) .
$$

The non-local interaction, i.e., the kernel $G(x)$ is adopted to be symmetric and normalized in the domain of interest $\mathcal{D}$, we use a square kernel defined as

$$
G(x)=\frac{1}{2 w}[\Theta(w-x) \Theta(w+x)],
$$

where $\Theta(x)=0$ if $x<0$ and $\Theta(x)=1$ if $x>0$, is the step function. Thus the limit $w \rightarrow 0$ reproduces a local interaction, and the limit $w \rightarrow L$ represents a non-local interaction in the complete domain $\mathcal{D}$. In [6] several types of kernels, and their analytical properties are presented.

The deterministic version of the model, Eq.(2.1) with $\epsilon=0$, has two homogeneous steady states: $\{0, a / b\}$. In the local case those values constitute the unstable and stable fixed points respectively; note that the non-local Fisher's model is non-variational. For the non-local case we are interested mainly in the instability that occurs with the non-zero state, i.e., $u_{0}=a / b$. This instability can be understood doing a linear analysis around $u_{0}$, see Eq.(3.5), and its appearing depends on the growth parameter $a$ the diffusion constant $D$ and the Fourier transformation of the non-local kernel $G(x)$, these characteristics are analyzed in detail in the following sections. Then, for a given set of parameters, see Eq.(3.6), the uniform initial condition $u_{0}$ becomes unstable, so due to fluctuations, the dynamics ends in a patterned stable solution. We show the comparison between deterministic vs. stochastic evolutions in Figure 1. In this figure one realization of the stochastic dynamics, Eq.(2.1), is shown in the course of time. In addition 


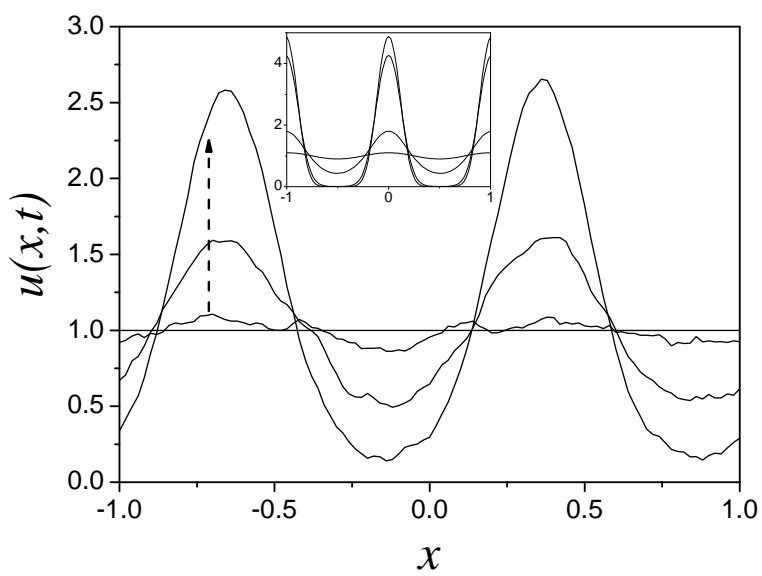

FIgURE 1. Typical stochastic evolution of the field $u(x, t)$. The initial condition is $u(x, 0) \equiv u_{0}=1$ and the evolution follows Eq.(2.1) with $\epsilon=10^{-2}$. The physical parameters $(a, b, D, w, L)$ are chosen in such a way the initial condition is unstable (see Tables I and II). The arrow shows the amplitude of the stochastic evolution at three different times: $t=(10,20,25)$, i.e., evolving from the uniform toward the patterned state. The inset shows the time-evolution to the final state from a pure deterministic solution with initial condition: $u(x, 0)=1+\sqrt{\epsilon} \cos \left(k_{e} x\right)$ for the set of times: $t=$ $(10,20,30)$.

in the inset of this figure we also show the evolution of a pure deterministic solution, for a similar set of times, and from the particular initial condition: $u(x, 0)=a / b+\sqrt{\epsilon} \cos \left(k_{e} x\right)$ (the uniform solution plus and small Fourier perturbation, see next section for the definition of $k_{e}$ ). As can be seen the attractor is almost reached (from this deterministic evolution) for a time around $t=30$. Therefore the important point in the description of the pattern formation is to investigate its transient stochastic dynamics. In the next sections we will be interested in the analytical description of the Mean First Passage Time (MFPT) leaving the uniform state to the final patterned stable solution. To do this we will introduce a multiscale perturbation expansion and used the stochastic path perturbation approach to tackle the evolution of Eq.(2.1).

\section{Fourier analysis}

As mentioned, in the present analysis we assume periodic boundary conditions in the interval $\mathcal{D} \equiv[-1,1]$, i.e., we use a domain size $L=1$. In order to study the transition from a uniform stationary state to a patterned one, we decompose Eq.(2.1) using a discrete Fourier transformation, as follows

$$
\begin{aligned}
u(x, t) & =\sum_{n=-\infty}^{\infty} A_{n}(t) \exp \left(i k_{n} x\right) \\
\xi(x, t) & =\sum_{n=-\infty}^{\infty} \xi_{n}(t) \exp \left(i k_{n} x\right) \\
G(x) & =\sum_{n=-\infty}^{\infty} G_{n} \exp \left(i k_{n} x\right),
\end{aligned}
$$


here $k_{n}=n \pi, n=0, \pm 1, \pm 2, \pm 3 \ldots$ and $\int_{-1}^{1} G(x) d x=1$. Introducing these series into Eq.(2.1) and using that

$$
\int_{-1}^{1} e^{i(m+n) \pi x} d x=2 \delta_{m+n, 0}
$$

we arrive at

$$
\begin{aligned}
\partial_{t} \sum_{n=-\infty}^{\infty} A_{n}(t) e^{i k_{n} x}= & \sum_{n=-\infty}^{\infty}\left(D\left(i k_{n}\right)^{2}+a\right) A_{n}(t) e^{i k_{n} x} \\
& -2 b\left(\sum_{m=-\infty}^{\infty} A_{m}(t) e^{i k_{m} x}\right)\left(\sum_{n=-\infty}^{\infty} G_{n} A_{n}(t) e^{i k_{n} x}\right) \\
& +\sqrt{\epsilon} \sum_{n=-\infty}^{\infty} \xi_{n}(t) e^{i k_{n} x}
\end{aligned}
$$

then using orthogonality of Fourier series we can write the following infinite set of coupled Fourier modes

$$
\frac{d A_{n}}{d t}=\left(-D k_{n}^{2}+a\right) A_{n}-2 b \sum_{l=-\infty}^{l=\infty} A_{n-l} A_{l} G_{l}+\sqrt{\epsilon} \xi_{n}(t), \quad\left\langle\xi_{m}\left(t^{\prime}\right) \xi_{n}(t)\right\rangle=\delta_{m+n} \delta\left(t-t^{\prime}\right)
$$

Introducing the usual linear stability analysis: $u=u_{0}+u_{1}$ with $u_{0}=a / b$ and $u_{1}=e^{\varphi t}\left(\sum_{n=-\infty}^{\infty} A_{n} e^{i k_{n} x}\right)$ into the deterministic part of Eq.(2.1) we get

$$
\begin{aligned}
\partial_{t} u_{1} & =D \partial_{x}^{2} u_{1}+a u_{1}-b u_{1} u_{0}-b u_{0} \int_{\mathcal{D}} u_{1}\left(x-x^{\prime}, t\right) G\left(x^{\prime}\right) d x^{\prime} \\
& =D \partial_{x}^{2} u_{1}+a u_{1}-b u_{1} u_{0}-2 b u_{0}\left(\sum_{n=-\infty}^{\infty} G_{n} A_{n} e^{\varphi t} e^{i k_{n} x}\right) .
\end{aligned}
$$

Therefore the homogenous state $u_{0}=a / b$ is unstable under small perturbations of the form

$$
u(x, t)=a / b+e^{\varphi t+i k_{n} x}
$$

if

$$
\varphi=-D k_{n}^{2}-2 a G_{n}>0 .
$$

For the particular kernel we use in the present work, Eq.(2.2), the dispersion relation $\varphi \equiv \varphi\left(k_{n}\right)$ is shown in Figure 2. Note that any typical length scale characterizing an abrupt condition for the kernel $G(x)$ (cut-off in the range of non-local interaction) appears in the final expression of the Fourier transformation $G_{n}$. As discussed in detail in [6] an interesting characteristic of this non-local dynamics is the appearance of a non-trivial unstable mode, as illustrated in Figure 1. In Tables I and II we show the corresponding numerical values of the parameters we have used.

Therefore depending on the physical parameters of the system new scenarios may appear, for example if the value of the diffusion coefficient changes (due to external agents) the stability of the homogeneous state $u_{0}=a / b$ may change, see Eq.(3.6) and Figure 2. In particular the situation when $\varphi\left(k_{e}\right)=0$ for a given value of $k_{e}$ may happen, leading therefore to a critical slowing down for the escape process. This critical case is much more complex to analyze because the instability turns to be non-linear. See also Eqs.(4.11)-(4.13) in the next section where we discuss the multiple scale dynamics of non-local Fisher's model in terms of a minimum coupling approximation. 
TABle 1. Parameters near criticality used.

\begin{tabular}{cc}
\hline \hline Physical parameters & Description \\
\hline \hline$a=1$ & Linear growth rate \\
$b=1$ & Nonlinear coupling parameter \\
$D=5 \times 10^{-4}$ & Diffusion coeficient \\
$L=1$ & Macroscopic size system \\
$w=0.7$ & Cut-off in the non-local interaction range \\
\hline
\end{tabular}

TABLE 2. Critical parameters.

\begin{tabular}{cc}
\hline \hline Physical parameters & Description \\
\hline \hline$G_{2}=\frac{1}{2} \frac{\sin 2 \pi w}{2 \pi w}$ & Fourier mode of the square non-local Kernel \\
$\varphi=-D(2 \pi)^{2}-2 a G_{2}>0$ & Phase in the small wave perturbation \\
\hline
\end{tabular}

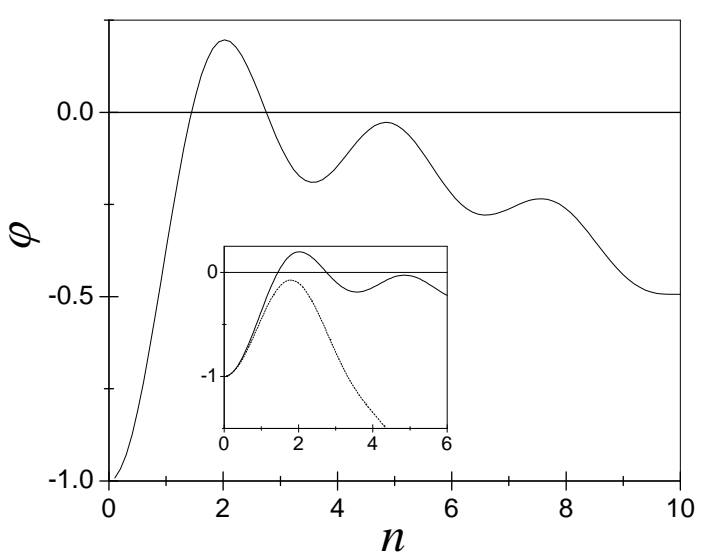

Figure 2. Dispersion relation $\varphi$ as a function of $n$. Here Eq. (3.6) is ploted for the parameter values shown in Tables I and II. Note that the unstable mode in this case corresponds to $n=2$. The dashed line in the inset is the result of using the same set of parameters but with the diffusion parameter $D=0.08$. For the last case (dashed line), the uniform state $u_{0}$ is stable.

\subsection{The Minimum Coupling Approximation}

From the coupled equations (3.2) we can make progress decreasing the dimension of the system near criticality, see appendix A. First consider the deterministic part of Eq.(3.2), then let us denote $u(x, 0)=$ $a / b>0$ the initial condition of the field, i.e., the uniform stationary state. Adopting the parameters (see Tables I and II) in such a way that there is only one Fourier unstable mode that we call $k_{e}$ in the present work (see Figure 2), we get in a short-time evolution

$$
A_{0}(\Delta t) \sim a / b+2 b A_{e}(0)^{2}\left|G_{e}\right| \Delta t,
$$

with $\left|A_{e}(0)\right| \ll a / b$, meaning that $A_{0}(t)$ grows linearly in time. While $A_{e}(t)$ grows exponentially

$$
A_{e}(\Delta t) \sim A_{e}(0) \exp [\varphi \Delta t] .
$$


We can therefore bring out what we call the Minimum Coupling Approximation (MCA), this approximation allows us to derive from Eq.(3.2) the following set of coupled deterministic equations (see appendix A)

$$
\begin{aligned}
& \frac{d A_{0}}{d t} \simeq a A_{0}-b A_{0}^{2}-2 b A_{e}^{2} G_{e} \equiv F\left(A_{0}, A_{e}\right) \\
& \frac{d A_{e}}{d t} \simeq a(e) A_{e}-b A_{e} A_{0}\left(1+2 G_{e}\right) \equiv Q\left(A_{0}, A_{e}\right),
\end{aligned}
$$

Here we have used $G_{0}=\frac{1}{2}$, the symmetries $a(n)=a(-n) \equiv\left(-D k_{n}^{2}+a\right)$, and $G_{n}=G_{-n}$ to conclude that $A_{e}=A_{-e}$. This means that Fisher's field is represented as

$$
u(x, t)=A_{0}+\sum_{n=1}^{\infty} 2 A_{n} \cos (n \pi x) .
$$

Interestingly the stationary state in the MCA can be found solving equations: $F\left(A_{0}, A_{e}\right)=$ $Q\left(A_{0}, A_{e}\right)=0$, then we obtain the solutions

$$
\begin{aligned}
A_{0} & =\frac{-D k_{e}^{2}+a}{b\left(1+2 G_{e}\right)} \\
A_{e}^{2} & =\frac{\left|1-D k_{e}^{2} / a\right| \varphi}{2\left(b^{2} / a\right)\left|G_{e}\right|\left(1+2 G_{e}\right)^{2}} .
\end{aligned}
$$

From the deterministic point of view [Eqs.(3.7)-(3.8)], when $\varphi>0$ the dominant time-evolution of the unstable mode $A_{e}(t)$ can be studied from the supercritical normal form:

$$
d z / d t=\varphi z-\beta z^{3}
$$

where $\beta \equiv 2 a\left(1-2\left|G_{e}\right|\right)(b / a)^{2}\left|G_{e}\right|$. Equation (3.12) can be obtained solving from (3.7) the stationary solution $A_{0}=A_{0}\left(A_{e}\right)$ and introducing this solution into the evolution Eq.(3.8) after invoking a Taylor expansion for small $A_{e}$ [5]. Note that from (3.12) the deterministic transient from $z(0)>0$ towards the final attractor $z(\infty)=\sqrt{\varphi / \beta}$ cannot be used to characterize a finite time-scale in the pattern formation. Therefore the statistical description of such a transient process is one of the main subjects in the study of nonequilibrium phenomena, where noise plays a crucial role in the dynamics of the system. This is the reason why the study of the MFPT turns to be of great importance in the analysis of pattern formation.

We remark that in the framework of the MCA it is possible to see, from Eqs.(3.10)-(3.11), that at the critical point $\left(\varphi \equiv\left(-D k_{e}^{2}+2 a\left|G_{e}\right|\right)=0\right)$ the stationary solutions are given by: $\left.A_{0}\right|_{\varphi \rightarrow 0} \rightarrow a / b$ and $\left.A_{e}\right|_{\varphi \rightarrow 0} \rightarrow 0$. So if we wish to calculate a finite value for $A_{e}(\infty)$ we should go beyond the MCA. On the other hand, the essential difficulty describing the relaxation from a state of marginal stability (when $\varphi=0$ ), is that there is no regime in which a linear approximation is meaningful. This subject will be clarified in the next section, see Eqs. (4.11) to (4.13).

\section{The Stochastic Multiscale Perturbation Expansion}

A better description of the complexity of the stochastic evolution can be obtained when introducing a stochastic multiscale expansion. In order to simplify this presentation let us introduce the main idea at the level of the MCA for the case $\varphi>0$.

The stochastic version of the set of coupled MCA Eqs.(3.7)-(3.8) can be written in the form 


$$
\begin{aligned}
& \frac{d A_{0}}{d t}=F\left(A_{0}, A_{e}\right)+\sqrt{\epsilon} \xi_{0}(t) \\
& \frac{d A_{e}}{d t}=Q\left(A_{0}, A_{e}\right)+\sqrt{\epsilon} \xi_{e}(t),
\end{aligned}
$$

where

$$
\left\langle\xi_{0}(t) \xi_{e}\left(t^{\prime}\right)\right\rangle=0,\left\langle\xi_{e}(t) \xi_{e}\left(t^{\prime}\right)\right\rangle=\left\langle\xi_{0}(t) \xi_{0}\left(t^{\prime}\right)\right\rangle=\delta\left(t-t^{\prime}\right) .
$$

If $\epsilon$ is a small parameter, we can introduce a stochastic multiscale perturbation expansion for the homogeneous mode, $A_{0}$, and the unstable one, $A_{e}$, in the form

$$
\begin{aligned}
& A_{0}(t)=A_{0}^{(0)}+\sqrt{\epsilon} x+\epsilon y+\cdots \\
& A_{e}(t)=\sqrt{\epsilon} W+\epsilon V+\cdots .
\end{aligned}
$$

Introducing Eqs.(4.4)-(4.5) into Eqs.(4.1)-(4.2), and collecting different orders in $\epsilon$ we obtain the multiple scale dynamics of the modes. For example for the homogeneous mode, up to $\mathcal{O}\left(\epsilon^{1}\right)$, we get

$$
\begin{aligned}
\mathcal{O}\left(\epsilon^{0}\right) & \Rightarrow A_{0}^{(0)}=a / b \\
\mathcal{O}\left(\epsilon^{1 / 2}\right) & \Rightarrow \frac{d x}{d t}=-a x+\xi_{0} \\
\mathcal{O}\left(\epsilon^{1}\right) & \Rightarrow \frac{d y}{d t}=-a y-b x^{2}-2 b G_{e} W^{2} .
\end{aligned}
$$

For the dynamics of the unstable mode we get

$$
\begin{aligned}
\mathcal{O}\left(\epsilon^{1 / 2}\right) & \Rightarrow \frac{d W}{d t}=\varphi W+\xi_{e} \\
\mathcal{O}\left(\epsilon^{1}\right) & \Rightarrow \frac{d V}{d t}=\varphi V-b\left(1+2 G_{e}\right) W x
\end{aligned}
$$

From this multiscale perturbation it is possible to see that in the supercritical case $\varphi>0$ the shorttime evolution is linear, therefore a Gaussian noise-perturbation dominates the escape process from the unstable state. However this (linear) simplification does not happen when $\varphi=0$, in fact at the critical point the relevant escape process is triggered by non-Gaussian fluctuations because the stochastic set of equations that dominates the escape processes are in this case:

$$
\begin{aligned}
\frac{d x}{d t} & =-a x+\xi_{0} \\
\frac{d W}{d t} & =\xi_{e} \\
\frac{d V}{d t} & =-b\left(1+2 G_{e}\right) W(t) x(t) .
\end{aligned}
$$

Due to the marginality of the unstable state, the order $\mathcal{O}\left(\epsilon^{1 / 2}\right)$ does not give a correct description for the escape time. Up to this order the MFPT would be the one associated to a pure diffusion problem which is not a good description of the escape from a marginal unstable state triggered by noise, so we have to go beyond the Gaussian description given by the process $W(t)$. To the next order $O\left(\epsilon^{1}\right)$, the stochastic evolution of $V(t)$ indeed controls the dominant contribution of the random escape time, but in this case the time evolution of $V(t)$ is governed by a "noise term" that is the product of a Wiener and an Orstein-Uhlenbeck process, so the stochastic process $V(t)$ would be non-Gaussian. 


\subsection{The Stochastic Path Perturbation Approach in the Supercritical Case}

In the small noise approximation $(\epsilon \ll 1)$ the Stochastic Path Perturbation Approach (SPPA) consists in obtaining information about the first passage time statistics without solving the Fokker-Planck equation. This is done by analyzing the stochastic realizations of the process under study when they are written in terms of Wiener paths [21].

Due to the linearity of the unstable evolution, the SPPA can easily be introduced working out the Wiener realization up to $\mathcal{O}\left(\epsilon^{1 / 2}\right)$ [22], on the other hand in the small noise approximation the first passage time statistics is independent of the saturation of the unstable mode [23]. Therefore in order to study the pattern formation in the system we will consider a threshold-value for the growth of the unstable mode $A_{e}(t)$ (to be able to calculate the MFPT without considering the saturation in the unstable mode $A_{e}(t)$, see Eq.(4.19)).

The supercritical case occurs when the factor $\varphi \equiv\left(-D k_{e}^{2}+2 a\left|G_{e}\right|\right)>0$, therefore the escape process of the unstable mode $A_{e}(t)$ is dominated by the $\mathcal{O}\left(\epsilon^{1 / 2}\right)$, i.e., the linear stochastic differential Eq.(4.9). Consistently the homogeneous mode is well described by Eqs.(4.6)-(4.7). We can interpret the stochastic multiscale dynamics in the following form: to $\mathcal{O}\left(\epsilon^{0}\right)$ the homogenous modes is the expected value $A_{0}^{(0)}=$ $a / b$, and to $\mathcal{O}\left(\epsilon^{1 / 2}\right)$ stochastic realizations $x(t)$ correspond to an Orstein-Uhlenbeck process which will lead to the saturation of the dispersion of the homogeneous mode: $A_{0}(t \gg a)=a / b+\sqrt{\epsilon} x(\infty)+\cdots$. Concerning the unstable mode, up to $\mathcal{O}\left(\epsilon^{1 / 2}\right)$ the realization $W(t)$ corresponds to an exponentially increasing stochastic process, therefore these realizations will lead to the dominant escape process toward the final attractor of the non-local Fisher equation. Using that $\varphi \equiv\left(-D k_{e}^{2}+2 a\left|G_{e}\right|\right)>0$, from Eqs.(4.7) and (4.9) we can write both stochastic realizations in the following form

$$
\begin{aligned}
x(t) & =\int_{0}^{t} \exp \left[-a\left(t-t^{\prime}\right)\right] \xi_{0}\left(t^{\prime}\right) d t^{\prime}, x(0)=0, t \geq 0 \\
W(t) & =\int_{0}^{t} \exp \left[\varphi\left(t-t^{\prime}\right)\right] \xi_{e}\left(t^{\prime}\right) d t^{\prime}, W(0)=0, t \geq 0 .
\end{aligned}
$$

Form expression (4.14) we note that for $t \rightarrow \infty$ the process $x(t)$ saturates to its stationary state, therefore we can introduce the notation $\Omega$ to characterize the random variable $x(\infty)=\Omega$, which, in addition from Eq.(4.7) can be seen to be characterized by the normal pdf

$$
P(\Omega)=\frac{\exp \left(\frac{-\Omega^{2}}{2 \sigma_{\Omega}^{2}}\right)}{\sqrt{2 \pi \sigma_{\Omega}^{2}}}, \sigma_{\Omega}^{2}=\frac{1}{2 a}, \Omega \in(-\infty, \infty) .
$$

On the other hand from (4.15), the stochastic process $W(t)$ can be written in the form

$$
\begin{aligned}
W(t) & =e^{\varphi t} \int_{0}^{t} \exp \left[-\varphi t^{\prime}\right] \xi_{e}\left(t^{\prime}\right) d t^{\prime} \\
& \equiv e^{\varphi t} \eta(t),
\end{aligned}
$$

where the process $\eta(t)$ fulfills the stochastic differential equation

$$
\frac{d \eta}{d t}=e^{-\varphi t} \xi_{e}(t), \eta(0)=0, t \geq 0
$$

Even more, it is possible to see that the stochastic process $\eta(t)$ also saturates for times $t \gg \varphi^{-1}$. Then the random variable $\eta(\infty) \equiv \eta$ is characterized by the normal pdf

$$
P(\eta)=\frac{\exp \left(\frac{-\eta^{2}}{2 \sigma_{\eta}^{2}}\right)}{\sqrt{2 \pi \sigma_{\eta}^{2}}}, \sigma_{\eta}^{2}=\frac{1}{2 \varphi}, \eta \in(-\infty, \infty) \equiv \mathcal{D}_{\eta} .
$$


Approximating $\eta(t) \sim \eta(\infty)$ in Eq.(4.17) we can extract the escape times $t_{e}$ inverting a random mapping (this is the core of the SPPA for linear unstable states), i.e., we can study the random escape times $t_{e}$ form a random transformation law $\eta \rightarrow t_{e}$ (into a suitable support to assure the positivity of $t_{e}$ ). To see this fact we first define $t_{e} \geq 0$ as the time when the stochastic process $W(t)$ reach a threshold value $W_{e}$,

$$
W_{e}^{2}=W\left(t_{e}\right)^{2} \simeq e^{2 \varphi t_{e}} \eta(\infty)^{2}=\eta^{2} \exp \left(2 \varphi t_{e}\right),
$$

then we can solve from (4.19) the random escape time as a function of the threshold $W_{e}$

$$
\begin{aligned}
t_{e} & =\frac{1}{2 \varphi} \ln \left(\frac{W_{e}}{\eta}\right)^{2}, \quad \frac{W_{e}}{\eta} \geq 1 \\
\eta & \Rightarrow \text { Normal distributed, see Eq.(4.18). }
\end{aligned}
$$

Now using the scaling Eq.(4.5) we write $W_{e}=A_{e} / \sqrt{\epsilon}$. Then the random mapping we were looking for is

$$
t_{e}=\frac{1}{2 \varphi} \ln \left(\frac{A_{e}^{2}}{\eta^{2} \epsilon}\right)
$$

Here $A_{e}$ will be considered as a threshold-value to be taken into account to characterize the pattern formation, i.e., the transition from the unstable state to the patterned state.

An asymptotic value $A_{e}(\infty)$ can be estimated from the stationary solution of the deterministic MCA, Eq.(3.11). Alternatively, $A_{e}(\infty)$ can be approximated from the attractor of the normal form (3.12) associated to the explosive mode $A_{e}(t)$, i.e., $A_{e}(\infty)=\sqrt{\varphi / \beta}$, where $\varphi$ is the phase of the Fourier mode and $\beta \equiv 2 a\left(1-2\left|G_{e}\right|\right)(b / a)^{2}\left|G_{e}\right|$.

\subsubsection{The mean first passage time}

We can calculate the MFPT taking the average on Eq.(4.20) to obtain

$$
\left\langle t_{e}\right\rangle=\frac{1}{2 \varphi}\left\langle\ln \frac{A_{e}^{2}}{\eta^{2} \epsilon}\right\rangle
$$

so using a nondimensional time we get

$$
\left\langle\tau_{e}\right\rangle=\ln (K)+\frac{\mathcal{E}+\ln 4}{2 \operatorname{erf}(K)}, K \gg 1, \tau_{e}=\varphi t_{e},
$$

where $\mathcal{E}$ is the Euler constant and $K=A_{e} \sqrt{\frac{\varphi}{\epsilon}}$ is the universal parameter of the system, see appendix B.

\subsubsection{The first passage time distribution}

Finally, the pdf for the escape times, i.e., the first passage time distribution $\mathcal{P}\left(t_{e}\right)$, can be obtained from the theorem of the transformation of random variables:

$$
\mathcal{P}\left(t_{e}\right)=\int_{\mathcal{D}_{\eta}} \delta\left(t_{e}-\frac{1}{2 \varphi} \ln \frac{A_{e}^{2}}{\eta^{2} \epsilon}\right) P(\eta) d \eta, \quad t_{e} \geq 0
$$

i.e., after some algebra we get (see appendix B)

$$
\mathcal{P}\left(\tau_{e}\right)=\frac{2 K}{\operatorname{erf}(K) \sqrt{\pi}} \exp \left[-\tau_{e}-K^{2} \exp \left(-2 \tau_{e}\right)\right], K=A_{e} \sqrt{\frac{\varphi}{\epsilon}}, \tau_{e}=\varphi t_{e} .
$$

These results could have been obtained using the SPPA without invoking a multiple scale analysis [24]. Nevertheless the stochastic multiple scaling analysis, Eqs.(4.4)-(4.5), is necessary if we want to tackle the escape process at the critical point $\varphi=0$, or if the noise appears in some physical parameter of the system 
like for example in $a, b$, or $D$ leading therefore to a multiplicative stochastic partial integro-differential equation, instead of the additive model given in Eq.(2.1). Work along these lines will be presented elsewhere.

As can be seen in Figure 3 the comparison with the Monte Carlo simulations show a good agreement with (4.23). The parameters that we have used in the simulations of Eq.(2.1) are shown in Tables I \& II, and the noise intensity was $\epsilon=\left\{10^{-2}, 10^{-4}\right\}$. In according with these parameters the unstable initial condition of the field was chosen $u(x, 0)=1$. As can be seen from Figure 1 a particular stochastic evolution shows the transient to the final steady state (compare with deterministic evolution shown in the inset). Thus in order to characterize the pattern formation in the system we use a threshold $\Delta$ for each stochastic realization: $\Delta \equiv \max \left[u\left(x, t_{e}\right)\right]-\min \left[u\left(x, t_{e}\right)\right]=1$. Therefore we can use the theoretical pdf for the escape time, Eq.(4.23), using a threshold-value $A_{e} \simeq 0.5$.

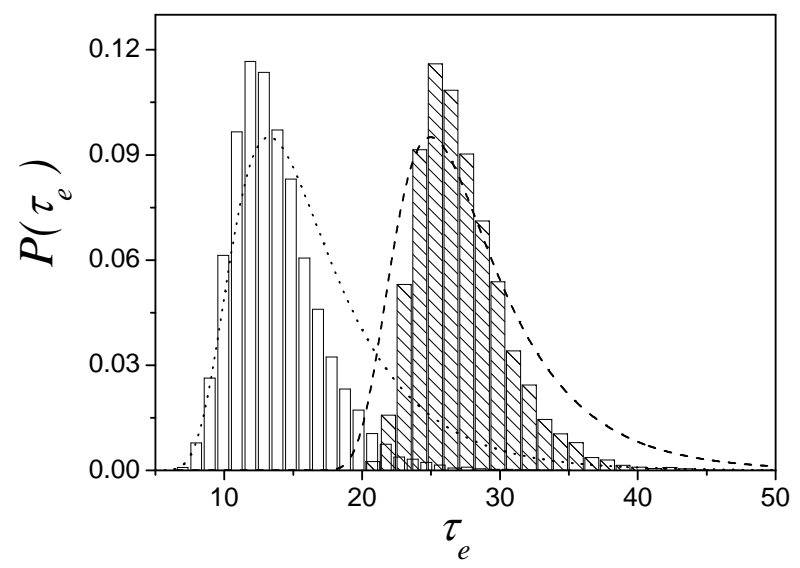

FIGURE 3. Escape time probability distribution, for the supercritical case, from Eq.(4.23) as a function of the nondimensional time $\tau_{e}$. The value of the parameters that we have used are shown in Tables I and II (thus $\varphi \simeq 0.19649, \beta \simeq 0.16947$ and so $A_{e}(\infty) \sim$ $\sqrt{\varphi / \beta} \simeq 1.076$ ). Dotted line: theoretical distributions for $\epsilon=10^{-2}$, while the dashed line corresponds to $\epsilon=10^{-4}$. Bar plots correspond to the simulations of Eq.(2.1) for each corresponding value of $\epsilon$.

\section{Conclusions}

In this work we presented a general approach to tackle the problem of the characterization of the Mean First Passage Time from an initial homogenous unstable state towards a final patterned stable attractor. In particular we applied this general approach when these states are associated to an stochastic integrodifferential spacial dynamics as in a Fisher-like equation. The general theory is based on the Stochastic Path Perturbation Approach with the implementation of the Minimum Coupling Approximation that allowed us to study the random escape times from the initial unstable state.

In the present paper we introduced, in addition, a stochastic multiple scale analysis which is a fundamental tool that unable us to undertake linearly unstable states, as well as nonlinear instabilities where the fluctuation responsible for the escape process cannot be described in terms of Gaussian statistics, see Eqs.(4.11)-(4.13). This critical case, $\varphi\left(k_{e}\right)=0$, may happen when some parameters are modified because of external reasons, for example, the diffusion coefficient may change altering the mobility of the 
species, see Figure 2. On the other hand, if the noise appears in some physical parameter of the evolution dynamics, for example the growth rate changes in the form $a \rightarrow a+\xi(x, t)$, the stochastic problem turns to be of multiplicative character, which is different to the additive problem presented in Eq.(2.1). These cases can only be tackled properly using the stochastic multiscale perturbation approach that we have introduced in the present work, these subjects under current investigation.

We are confident that the present theoretical approach to solve the First Passage Time Statistics, may help the general understanding of the pattern formation in complex systems, where diffusion (extended process) versus non-local interactions (with kernel and range interactions) play an important role in the description of real systems. The present stochastic multiple scale approach may also help to solve a different but related problem: the study of distributed time-delayed 0-dimensional dynamical systems that can be of interest in biological models $[25,26]$.

Acknowledgements. M.O.C. thanks grant from SECTyP, Universidad Nacional de Cuyo, Argentina, and grant PIP 90100290 (2010-12) from CONICET, Argentina. M.A.F. thanks CONICYT Project: Anillo en Complejidad Social SOC-1101 and FONDECYT 1140278.

\section{Appendix A: Reduction to the Minimum Coupling Approximation}

When there is only one unstable Fourier mode $A_{e}$ the deterministic part of the set of equations (3.2) can be written in a more friendly way separating the dynamics of the homogeneous and the unstable modes, as well as neglecting the dynamics of the rest of the modes $A_{l}, \forall l \neq \pm e, 0$ :

$$
\begin{aligned}
\frac{d A_{0}}{d t} & =\left(a-b A_{0}\right) A_{0}-2 b A_{e} A_{-e} G_{e}+\sum_{l \neq 0, e} \mathcal{O}\left(A_{l} A_{-l}\right) \\
\frac{d A_{e}}{d t} & =\left[a(e)-b A_{0}\left(1+2 G_{e}\right)\right] A_{e}-\sum_{l \neq 0, e} \mathcal{O}\left(A_{e-l} A_{-l}\right) \\
\frac{d A_{-e}}{d t} & =\left[a(-e)-b A_{0}\left(1+2 G_{-e}\right)\right] A_{-e}-\sum_{l \neq 0,-e} \mathcal{O}\left(A_{-e-l} A_{-l}\right) .
\end{aligned}
$$

In the symmetrical case, i.e., when $G_{n}=G_{-n}$ and noting that $a(n)=a(-n) \equiv\left(-D k_{n}^{2}+a\right)$, from Eqs.(5.1)-(5.3) it can proved that $A_{n}=A_{-n}, \forall n$, therefore we can restrict the Fourier modes to a cosine expansion $u(x, t) \simeq A_{0}+\sum_{n=1}^{\infty} 2 A_{n} \cos (n \pi x)$, or what is equivalent to consider the deterministic dynamics in the form

$$
\begin{aligned}
\frac{d A_{0}}{d t} & \simeq\left(a-b A_{0}\right) A_{0}-2 b A_{e}^{2} G_{e}, \\
\frac{d A_{e}}{d t} & \simeq\left[a(e)-b A_{0}\left(1+2 G_{e}\right)\right] A_{e} .
\end{aligned}
$$

Here we have neglected contributions of the form $O\left(A_{l} A_{-l}\right), \forall l \neq e, 0$, this approximation gives what we have called the Minimum Coupling Approximation [5,24].

The stationary states of Eqs.(5.4)-(5.5) are characterized by equations:

$$
\begin{aligned}
\left(a-b A_{0}\right) A_{0} & =2 b A_{e}^{2} G_{e} \\
a(e) & =b A_{0}\left(1+2 G_{e}\right), A_{e} \neq 0,
\end{aligned}
$$

and their solutions are the ones presented in Eqs. (3.10) and (3.11). They represent the nonlinear structure of the pattern formation in the context of the MCA, i.e.:

$$
u(x, t=\infty) \simeq A_{0}(\infty)+2 A_{e}(\infty) \cos \left(k_{e} x\right),
$$

which of course is only an approximation to the true stationary state of Eq.(2.1), see inset of Figure 1. 


\section{Appendix B: The first passage time distribution}

Using the random mapping (4.20) and the normal distribution Eq.(4.18) we can calculate all the moments: $\left\langle t_{e}^{n}\right\rangle$, or the pdf (4.23). Introducing the Jacobian of the transformation in Eq.(4.22) we can write

$$
\begin{aligned}
\mathcal{P}\left(t_{e}\right) & =\int_{\mathcal{D}_{\eta}} \delta\left(t_{e}-t(\eta)\right) P(\eta) d \eta, t(\eta)=\frac{1}{2 \varphi} \ln \frac{A_{e}^{2}}{\eta^{2} \epsilon} \geq 0 \\
& =\mathcal{N} \int_{0}^{A_{e} / \sqrt{\epsilon}} \delta\left(\eta-\eta_{e}\right) P(\eta) \frac{d \eta}{\left|\frac{d t(\eta)}{d \eta}\right|_{\eta=\eta_{e}}}, \eta_{e}=\frac{A_{e}}{\sqrt{\epsilon}} e^{-\varphi t_{e}} \\
& =\mathcal{N} P\left(\eta_{e}\right)\left|\frac{d \eta}{d t_{e}}\right|_{\eta_{e}} \\
& =\mathcal{N} \frac{\exp \left(\frac{-1}{2 \epsilon \sigma^{2}} A_{e}^{2} e^{-2 \varphi t_{e}}\right)}{\sqrt{2 \pi \sigma^{2}}} \frac{A_{e} \varphi e^{-\varphi t_{e}}}{\sqrt{\epsilon}}, \sigma^{2}=\frac{1}{2 \varphi} .
\end{aligned}
$$

Introducing the nondimensional time $\tau_{e}=\varphi t_{e}$ the normalization constant $N$ can be calculated as

$$
\begin{aligned}
1 & =\int_{0}^{\infty} \mathcal{P}\left(\tau_{e}\right) d \tau_{e} \\
& =\frac{\mathcal{N}}{\sqrt{\pi}} \int_{0}^{\infty} A_{e} \sqrt{\frac{\varphi}{\epsilon}} e^{-\tau_{e}} \exp \left(-\frac{\varphi A_{e}^{2}}{\epsilon} e^{-2 \tau_{e}}\right) d \tau_{e} .
\end{aligned}
$$

Defining the auxiliary constant $K=A_{e} \sqrt{\frac{\varphi}{\epsilon}}$ and introducing the change of variable $x^{2}=K^{2} e^{-2 \tau_{e}}$ we get

$$
\begin{aligned}
1 & =\frac{\mathcal{N}}{\sqrt{\pi}} \int_{0}^{\infty} \exp \left(-\tau_{e}-K^{2} e^{-2 \tau_{e}}\right) d t_{e} \\
& =\frac{\mathcal{N}}{\sqrt{\pi}} \int_{0}^{K} e^{-x^{2}} d x \\
& =\frac{\mathcal{N}}{2} \operatorname{erf}(K) .
\end{aligned}
$$

Therefore the pdf can be written as

$$
\mathcal{P}\left(\tau_{e}\right)=\frac{2 K}{\sqrt{\pi} \operatorname{erf}(K)} \exp \left(-\tau_{e}-K^{2} e^{-2 \tau_{e}}\right), \quad K=A_{e} \sqrt{\frac{\varphi}{\epsilon}}, \quad \tau_{e}=\varphi t_{e},
$$

which is the result given in (4.23). The MFPT follows as: $\left\langle\tau_{e}\right\rangle=\int_{0}^{\infty} \tau_{e} P\left(\tau_{e}\right) d \tau_{e}$, which is the result given in Eq.(4.21).

\section{References}

[1] A. Mogilner, L. Edelstein-Keshet. A non-local model for a swarm. J. Math. Biol., 38 (1999), no. 6, 534-570.

[2] A. Mogilner, L. Edelstein-Keshet, L. Bent, A. Spiros. Mutual interactions, potentials, and individual distance in a social aggregation. J. Math. Biol., 47 (2003), no. 4, 353-389.

[3] J.D. Murray. Mathematical Biology, Vol. 1 and Vol. 2. Third Edition, Barcelona, Springer, Berlin, 2007.

[4] E. Hernandez-Garcia, C. Lopez. Clustering, advection, and pattern formation in a model of population dynamics with neighborhood-dependent rates. Physical Review E, 70 (2004), no. 1, 016216.

[5] M.A. Fuentes, M.N. Kuperman, V.M. Kenkre. Nonlocal Interaction Effects on Pattern Formation in Population Dynamics. Physical Review Letters, 91 (2003), no. 15, 1581041.

[6] M.A. Fuentes, M.N. Kuperman, V.M. Kenkre. Analytical Considerations in the Study of Spatial Patterns Arising from Nonlocal Interaction Effects. The Journal of Physical Chemistry B, 108 (2004), no. 29, 10505-10508.

[7] E. Ben-Jacob, I. Cohen, H. Levine. Cooperative self-organization of microorganisms. Advances in Physics, 49 (2000), no. $4,395-554$. 
[8] M.G. Clerc, D. Escaff, V.M. Kenkre. Pattern and localized structures in population dynamics. Physical Review E, 72 (2005), no. 5, 056217.

[9] I. Demin, V. Volpert. Existence of waves for a nonlocal reaction-diffusion equation. Mathematical Modelling of Natural Phenomena, 5 (2010), no. 5, 80-101.

[10] B.L. Segal, V.A. Volpert, A. Bayliss. Pattern formation in a model of competing populations with nonlocal interactions. Physica D: Nonlinear Phenomena, 253 (2013), 12-22.

[11] J.K. Hale, J.K. Kocak. Dynamics and Bifurcations. Texts in Applied Mathematics. Springer-Verlag. Corrected Edition, 1996.

[12] J. Guckenheimer, P. Holmes. Nonlinear Oscillations, Dynamical Systems and Bifurcations of Vector Fields. Applied Mathematical Sciences. Springer. 1st ed. 1983. Corr. 6th Edition, 2002.

[13] P.C. Bressloff, M.A. Webber. Front propagation in stochastic neural fields. SIAM Journal on Applied Dynamical Systems, 11 (2012), no. 2, 708-740.

[14] C. Kuehn, M.G. Riedler. Large deviations for nonlocal stochastic neural fields. J. Math. Neuroscience, 4 (2014), no. 1, $1-33$.

[15] V. Volterra. Variations and fluctuations of the number of individuals in animal species living together. J. Cons. Int. Explor. Mer, 3 (1928), no. 1, 3-51.

[16] R.A. Fisher. The wave of advance of advantageous genes. Annals of Eugenics, 7 (1937), no. 4, 355-369.

[17] M.O. Cáceres. Passage Time Statistics in Exponential Distributed Time-Delay Models: Noisy Asymptotic Dynamics. Journal of Statistical Physics, 156 (2014), no. 1, 94-118.

[18] N.G. van Kampen. Stochastic Processes in Physics and Chemistry, 2nd ed. North Holland, Amsterdam, 1992.

[19] M.O. Cáceres. Elementos de estadistica de no equilibrio y sus aplicaciones al transporte en medios desordenados. In Spanish, Reverté S.A., Barcelona, 2003.

[20] J. Garcia-Ojalvo, J. M. Sancho. Noise in Spatially Extended, Systems. Springer, Berlin, 2010.

[21] P. Colet, F. de Pasquale, M.O. Cáceres, M. San Miguel. Theory for relaxation at a subcritical pitchfork bifurcation. Physical Review A, 41 (1990), no. 4, 1901.

[22] M.O. Cáceres. Passage Time Statistics in a Stochastic Verhulst Model. Journal of Statistical Physics, 132 (2008), no. $3,487-500$.

[23] M.O. Caceres, Ch. D. R. Rojas. Exponential distributed time-delay nonlinear models: Monte Carlo simulations. Physica A, 409 (2014), 61-70.

[24] M.A. Fuentes, M.O. Cáceres. First Passage Time on Pattern Formation in a Non-local Fisher Population Dynamics. Central European Journal of Physics, 11 (2013), no. 12, 1623-1628.

[25] M.O. Cáceres. Time-delayed coupled logistic capacity model in population dynamics, Physical Review E, 90 (2014), no. $2,022137$.

[26] S. Gonçalves, G. Abramson, M.F.C. Gomes. Oscillations in SIRS model with distributed delays. The European Physical Journal B, 81 (2011), no. 3, 363-371. 